\title{
Reference data for the adsorption of benzene on carbon materials
}

The adsorption of benzene at $298 \mathrm{~K}$ on carbon blacks and microporous carbons, including Carbosieve, Takeda molecular sieves, Maxsorb superactivated carbons and an activated charcoal cloth, has been studied. It is shown that although it is not possible to define a universal standard benzene isotherm, reference isotherms can be defined and used successfully to determine micropore volumes and external surface areas of microporous carbon adsorbents as well as to obtain estimates of surface areas of carbon blacks.

Corresponding author: peter@uevora.pt

doi:10.1016/S0008-6223(99)00129-3 